\title{
A New Method for Analysis of Biomolecules Using the BSM-SG Atomic Models
}

\author{
Stoyan Sarg Sargoytchev* \\ World Institute for Scientific Exploration, York University, Canada
}

\begin{abstract}
Biomolecules and particularly proteins and DNA exhibit some mysterious features that cannot find satisfactory explanation by quantum mechanical modes of atoms. One of them, known as a Levinthal's paradox, is the ability to preserve their complex three-dimensional structure in appropriate environments. Another one is that they possess some unknown energy mechanism. The Basic Structures of Matter Supergravitation Unified Theory (BSM-SG) allows uncovering the real physical structures of the elementary particles and their spatial arrangement in atomic nuclei. The resulting physical models of the atoms are characterized by the same interaction energies as the quantum mechanical models, while the structure of the elementary particles influence their spatial arrangement in the nuclei. The resulting atomic models with fully identifiable parameters and angular positions of the quantum orbits permit studying the physical conditions behind the structural and bonding restrictions of the atoms connected in molecules. A new method for a theoretical analysis of biomolecules is proposed. The analysis of a DNA molecule leads to formulation of hypotheses about the energy storage mechanism in DNA and its role in the cell cycle synchronization. This permits shedding a light on the DNA feature known as a C-value paradox. The analysis of a tRNA molecule leads to formulation of a hypothesis about a binary decoding mechanism behind the 20 flavors of the complex aminoacyle-tRNA synthetases - tRNA, known as a paradox.
\end{abstract}

Keywords: C-Value paradox; Levinthal's paradox; DNA research; Structure of biomolecules; Structural chemistry; Biophysics; Biochemistry

\section{Introduction}

Despite the huge number of possible configurations of atoms in proteins, according to the Quantum mechanics (QM), they fold reliably and quickly to their native state. This effect is known in the biochemistry as Levinthal's paradox. If we consider only the Quantum mechanical approach, a protein molecule made from 2,000 atoms, for example, should possess an astronomical number of degrees of freedom. In reality this number is drastically reduced by some strong structural restrictions, such as bond lengths, bond angles and rotation restrictions. The complex secondary and tertiary structures of biomolecules provide indications of additional restrictions with weaker strength but responsible for their complex shapes.

The BSM Supergravitation Unified Theory (BSM-SG), based on a new approach [1] is presented in a monograph [2]. It unveils a space micro-curvature around the superdense atomic nuclei that distorts significantly the real dimensions of the elementary particles and atomic nuclei. This is the reason that Quantum mechanics works only with energy levels. The atomic models derived in the BSM-SG theory [2] show that elementary particles and atomic nuclei are non-spherical, while the atoms posses the same energy levels. They have rich 3D structures defining the spatial arrangements of the electronic orbits, which determine the angular positions of the chemical bonds. The 3D structure of the stable elementary particles and atomic nuclei are presented in the Atlas of Atomic Nuclear Structures (ANS) [3], which is an Appendix of the BSM-SG monograph [2]. The purpose of this article is to suggest a new analytical approach for studying the properties of biomolecules.

\section{General considerations}

The three-dimensional structures of many biomolecules and their atomic compositions are now well known. In these structures, the individual atoms are identified as nodes with known coordinates.
The angular coordinates of their chemical bonds are also known. This information is sufficient for an analysis permitting replacement of the nodes in the 3D structure of any large molecule with the physical models of the atoms according to the BSM-SG theory. The application of the BSM-SG atomic models, for example, provides the possibility to identify the positions of all electronic orbits including also those responsible for the chemical bonds. The BSM-SG atomic models permits modelling and analysis in a real sub-nanometric scale, so the conditions for possible interactions, modifications and energy transfer could be analysed at the atomic level [4].

\section{Atomic ring structures in organic molecules}

Most of the organic molecules contain ring-like atomic structures. The molecule of benzene could be considered as a simple example of a ring structure. Figure 1 show the $3 \mathrm{D}$ molecular structure of aspirin where the ring structure of 6 carbon atoms is similar to the benzene molecule.

Figure 2 shows the same structure of aspirin by the BSM-SG's atomic models. The single atoms: Deuteron, Oxygen and Carbon and the size of the quantum orbit of the second subharmonic are shown in the box at the upper left corner. The valence protons (deuterons) of the oxygen atom are in fact in a plane perpendicular to the plane of EB bonded protons (deuterons) (see $\$ 8.68$, Chapter 8 of BSM-SG), but they are shown with reduced dimensions to imitate an oblique angle in

${ }^{*}$ Corresponding author: Stoyan Sarg Sargoytchev, Lassonde Bldg., 2039, York University, 4700 Keele Street, Toronto, ON M3J 1P3, Canada, Tel: +1 416-7362100; E-mail: stoyans@cse.yorku.ca

Received February 01, 2017; Accepted March 31, 2017; Published April 03, 2017

Citation: Sargoytchev SS (2017) A New Method for Analysis of Biomolecules Using the BSM-SG Atomic Models. J Biom Biostat 8: 339. doi:10.4172/21556180.1000339

Copyright: (c) 2017 Sargoytchev SS. This is an open-access article distributed under the terms of the Creative Commons Attribution License, which permits unrestricted use, distribution, and reproduction in any medium, provided the original author and source are credited. 
Citation: Sargoytchev SS (2017) A New Method for Analysis of Biomolecules Using the BSM-SG Atomic Models. J Biom Biostat 8: 339. doi:10.4172/2155-6180.1000339

Page 2 of 16

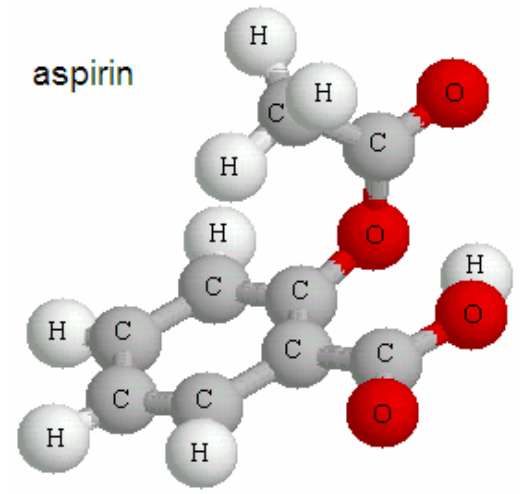

Figure 1: Three dimensional structure of the aspirin molecule (PDB file aspirin visualized by Chime software).

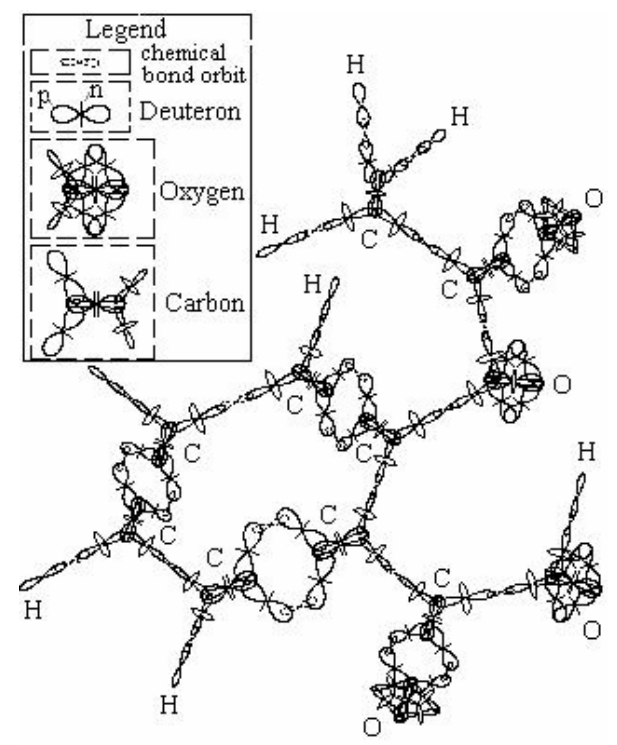

Figure 2: Three-dimensional structure of aspirin using the BSM-SG atomic models. Electronic orbits of chemical bonds are shown by dashed lines.

a $3 \mathrm{D}$ view. The same is true also for the valence protons of the carbon atom. In Figure 2, only the electronic orbits involved in the chemical bonds are shown. For molecules with known 3D structure and composition, the common positions of all electronic orbits with their equivalent orbital planes are identifiable [3]. It is clearly apparent that the $3 \mathrm{D}$ structure of the molecule is defined by the following conditions:

(a) a finite size of the involved atomic nuclei

(b) a restricted angular freedom of valence protons

(c) a finite orbital trace length defined by the quantum conditions of the circulating electron

(d) orbital interactions

(e) a QM spin of the electron (the motion direction of the electron relative to the proton twisting)

The QM mechanical models of atoms are mathematical models in which features (c), (d), and (e) are directly involved. The features (a) and (b) however are not apparent. In these mathematical models, some of the spatial and almost all angular restrictions are lost. Let us emphasize now the difference between the proposed BSM-SG models of atoms and molecules, from one hand and the QM models from the other:

QM model: the electrons participating in chemical bonds are orbiting around both point-like nuclei, i. e. they are not localised

BSM-SG model: the electrons involved in the chemical bonds do not orbit both nuclei and they are localised

QM model: the chemical bond lengths are calculated from the electron microscopy assuming the planetary atomic model in which the larger electron concentrations are centred around the point-like nucleus

BSM-SG model: the chemical bond lengths are not referenced to the center of the atomic nuclei.

QM model: The calculated length of the $\mathrm{C}=\mathrm{C}$ double valence bond is $1.34 \mathrm{~A}$ (angstrom), while for a single valence: $\mathrm{C}-\mathrm{C}$, it is $1.54 \mathrm{~A}$. However, these lengths show a small variation when the same ring-type groups are included in various biomolecules.

BSM-SG model: The length of single C-C bond may vary only in the subharmonic number of the quantum orbit, while the length in a double $\mathrm{C}=\mathrm{C}$ bond is dependent also on the angular positions of the valence protons. From the BSM-SG point of view, the single and double bond in benzene molecule, for example are not equal, because the is an angle between double bonds, defined by the nuclear configuration. This feature is quite important for uncovering some specific properties of the ring structures in biomolecules.

Ring structures are very abundant in many biomolecules and they are usually arranged in particular order along their chain. DNA and proteins contain a large number of ring structures. Figure 3 shows the spatial arrangement of the atomic ring structures in one part of b-type DNA. The positions of some $(\mathrm{O}+4 \mathrm{C})$ rings in the deoxyribose molecule that are involved in the helical backbone strands of DNA are indicated by arrows.

Figure 4 shows the atomic ring structure $(\mathrm{O}+4 \mathrm{C})$ of the DNA strand using BSM-SG atomic models.

The bonding deuterons (protons) involved in the ring structure practically have some small twisting. Following the proximity electrical field of the proton, the quantum orbit of single valence bond also have a very small twisting but it could be considered as flat. However, it may have some freedom of twisting for a single valence bond. This feature gives some freedom for the formation of ring structures from various

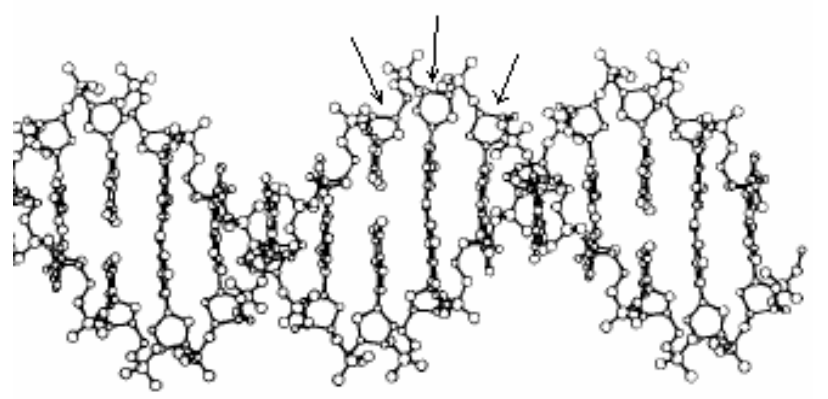

Figure 3: Part of DNA structure showing the positions of some $(\mathrm{O}+4 \mathrm{C})$ atomic rings. 


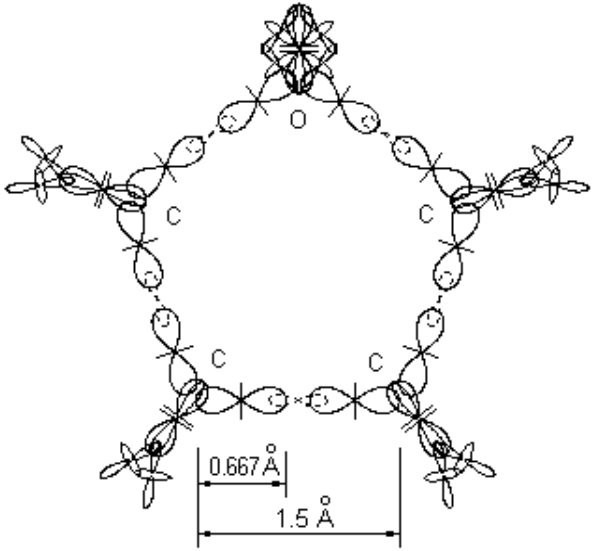

Figure 4: Atomic ring structure from the deoxyribose molecule involved in DNA strand.

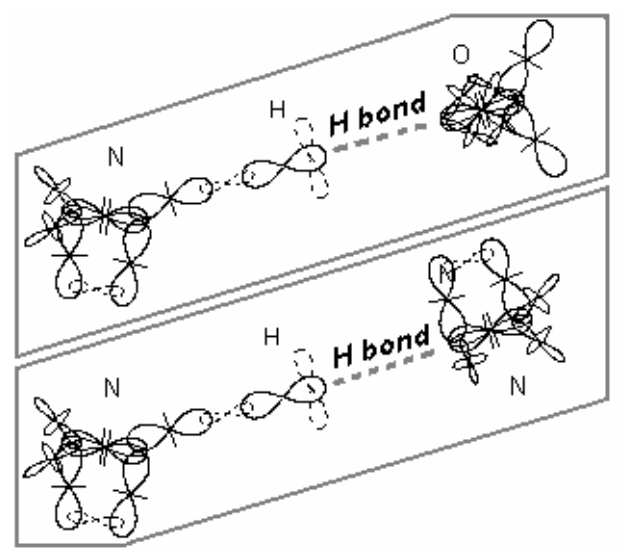

Figure 5: Two types of hydrogen bonds, denoted by straight dashed lines. Electronic orbits involved in the magnetic interactions are curves shown by dashed lines.

atoms. The rotational freedom of the single valence bonds, however, may be accompanied by some stiffness, which increases with the degree of orbital twisting. In the DNA molecule, some of the atoms of the ring structure are also connected to other external atoms. All these considerations explain why the ring structure $(\mathrm{O}+4 \mathrm{C})$ connected to the DNA strand is not flat but curved.

\section{Weak hydrogen bonds}

It is known that a weak hydrogen bond is possible between two atoms, one of which does not possess a free valence. The bond connection is a result of magnetic orbital interactions. In this case, the hydrogen bonds connecting the purines to pyramidines in DNA molecule are of two types:

$<\mathrm{N}-\mathrm{H} \ldots \mathrm{O}\rangle$ and $\langle\mathrm{N}-\mathrm{H} \ldots \mathrm{N}\rangle$, where the single valence electronic bond is denoted by "_" and the H-bond is denoted by "...". This is illustrated by Figure 5 .

BSM-SG theory allows us to find the possible orbital orientation for this type of bond. In a hydrogen bond of type N-H...O the plane of the electronic orbit of hydrogen appears almost parallel to the mutually oriented nuclear orbits of the lower electronic shell of the oxygen atom.
The orbital positions are defined by the arrangement of the protons in the atomic nucleus. [3]. In a hydrogen bond of type $\mathrm{N}-\mathrm{H}$...N, the plane of the electronic orbit of hydrogen is almost parallel to the equivalent planes of the two polar orbits of $\mathrm{N}$ in which two electrons are involved (Chapter 8 of BSM-SG) [3].

It is evident that the hydrogen bond is characterized by the following features:

- The connection is a result of mutual orbital orientation

- The H-bond requires a critical range of separation distance

- The H-bond allows rotational freedom within a limited angular range.

These three features permit the DNA molecule to possess excellent folding properties.

\section{Hypothesis of the energy storage mechanism in molecules possessing atomic ring structures}

It is well known from atomic spectra that only the alkali metals (Group I) and the positive atomic ions with a single valence electron possess atomic spectra that could be described by the Bohr atomic model. For elements with more than one valence electron, the principal quantum numbers exhibit more than one energy level, due to the spin orbital interactions. The signature of this feature is apparent from the Grotrian diagrams for atomic spectra. The spin-orbit interaction was discussed from a new point view in Chapter 8 of BSM-SG theory [3]. The analysis leads to the conclusion that after an orbiting electron is dropped to a lower quantum level, the pumped CL space energy is not fully emitted. Part of it is preserved by the atomic nucleus and redirected to the valence proton, whose quantum orbital plane is parallel to the orbital plane of the consideration. The physics of this effect is explainable if we consider the total energy balance including SG field of the nucleus (strong nuclear forces). The SG field controls the proton's proximity E-field distribution that defines the orbital conditions of the electron. Therefore, the redirected energy provides some shift of the energy levels, but the effect is stronger for the lower states closer to the ground state of the series (Balmer series has its own ground state, Chapter 7 of BSM-SG) [3]. The physical explanation of this effect allows us to make the conclusion that the released energy prior to formation of a photon is preferentially guided by the structures of protons (deuterons) held by the strong SG forces. Applying the same logic to the atomic ring structures, there must therefore be a guiding energy process between the atomic nuclei or protons involved in the ring. This consideration leads to the following conclusion:

In appropriate environments, the atomic ring structures in organic molecules may be able to store energy as an exited state rotating in the ring.

The effect of the rotating excited state is possible due to the consecutive re-excitation of the electrons in the adjacent quantum orbits involved in the valence bonding between the atoms connected in the ring. This effect is not apparent in the Quantum mechanical model, where the wave functions are complex envelopes around the entire nuclei of the involved atoms. However, it is a known fact that the bonding strengths between atoms involved in an atomic ring structure are stronger than between the same atoms when not participating in such structures.

Evidently, the rotating excited state in a ring structure could be obtained only for equal energy level differences. This view needs to 
consider excited states associated not only with the same valence bonds but also from single and second valence bonds as well. On the other hand, for a ring structure containing more than two bonds of the same valence, excited states may exist preferentially between the similar valence bonds. In the case of aspirin, for example, these conditions exist for three pairs of orbits of second valence and three pairs orbits of single valence bonds. If we also consider the fixed nuclear orbits (of lower electronic shells) of the atoms in the ring, then twelve polar electrons could be involved in a ring energy storage effect. Theoretically, they may store a much larger energy not only due to their number, but also due to the larger number of transitions with similar energies.

In appropriate environments, the stored energy in the ring structures of the biomolecules might have the following features:

- A stable cycle of an excited rotation state due to the stable finite time of a single excited state

- A possibility for interactions with properly oriented neighbouring ring structures in the instant between two consecutive excited states (conditions for synchronization between the rotation states of neighbouring rings)

\section{- A cascade type of energy transfer}

Many of the building blocks of the biomolecules or reagents contain atomic rings that we identify as a single or attached type. For example, Adenine ( 2 attached rings), Guanine (2 attached rings). Vitamin D contains one single and two attached rings. Alpha and Beta tubulins contain groups of: GDP (one single and two attached rings), GTP (one single and two attached rings), TAXOL (4 single rings). The steroid hormones usually contain four attached atomic rings. ATP, an important energy carrier in cells, contains one single and two attached rings. It is quite logical to consider that the rotating energy cycles in the attached rings are mutually dependant, so they might be synchronized. Then it is logical to expect that the attached rings may have an increased ability to hold stored energy.

\section{Hypothesis of energy flow through the chain structure of the biomolecule}

Energy flow in a DNA molecule and its effect on the higher order structural characteristics: The atomic ring structures in the long chain biomolecules appear to be arranged in some spatial order. In the molecule of DNA, for example, they are characterised by:

(a) a strong repeating order

(b) a strong orientation relative to the host strand of DNA

(c) a strong orientational order of the neighbouring rings along the helix

These well-known features are easily visualized when rotating the 3D structure of DNA (by programs such as: "Chime" "Rasmol", "Protein explorer" etc).

The idea of a cascade type of excited state transfer could be applied not only to an atomic ring structure but also to a long chain molecule built of repeatable atomic structures connected by electronic bonds. In this case, more complicated but mutually dependant mechanisms are involved. The following analysis tries to uncover these mechanisms.

Let us consider for this purpose one of the backbone strands of DNA. Figure 6 illustrates the connection path of the electronic bonds in the strand. Three important features are apparent for every repeating cascade:

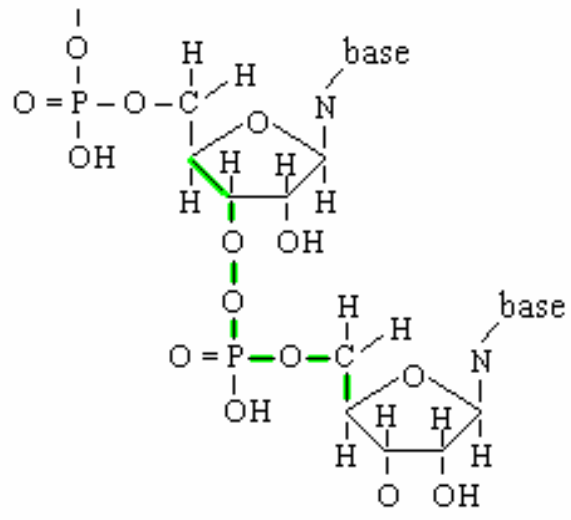

Figure 6: Bond connection path through a DNA strand. The connection path corresponding to one cascade of the nucleotide is denoted by a thick green line.

(d) the bond connection path is formed by deuterons or protons connected by electronic orbits

(e) the bond connection path passes through a $\mathrm{C}-\mathrm{C}$ bond from the $(\mathrm{O}+4 \mathrm{C})$ ring

(f) all bonds involved in the bond-connection path are single valence.

It is reasonable to expect that the long chain involving single valence bonds may provide conditions for cascade excited state transfer in one direction. The life-time of every excited state is determined by a quantum mechanical consideration - the life-time of the spontaneous emission. Keeping in mind the small distance between the neighbouring electronic bonds (of the order of 1-2 angstrom), the transfer time between two consecutive excited states (with the speed of light) could be ignored. Then the time dependence between the two energy processes (the cascade energy transfer and the energy rotation period in the ring) is easily obtained. The bonding path of one cascade contains six bonds in total, in which one $\mathrm{C}-\mathrm{C}$ bond from the $(\mathrm{O}+4 \mathrm{C})$ ring is included. This ring involves five bonds. Then the following condition holds:

$$
0 \leq\left(\mathrm{T}_{\mathrm{R}}-\mathrm{t}_{\mathrm{C}}\right) \leq \tau_{\mathrm{av}}
$$

where: $T_{R}$ - is a cycle period of the rotating state in the ring, $t_{c}$ - is a time interval for energy transfer in one cascade of the nucleotide, calculated from the sum of lifetimes of excited states in the bonds; $\tau_{\mathrm{av}}-$ is an average life-time of an excited state in a single bond.

Expression (1) means that the cascade transfer and the ring cycle are time-dependant, so they should have an appropriate phase synchronization. Additionally, all parameters of Equation (1) depend on temperature, but in a different way. This will impose a temperature range constraint for successful phase synchronization. Consequently: (g) the entire mechanism will work at an optimal temperature within a limited temperature range defined by the conditions of optimal phase between the cascade energy transfer and the ring energy cycle.

Let us analyse now the conditions that may support the tendency of unidirectional energy transfer. For this purpose we will consider a small portion of DNA. Ignoring the supercoiling in this case it could be regarded as a linear type of DNA. Let us take into account the following two structural features of Beta type DNA that might be related to the tendency of unidirectional energy transfer: 
- It is well known that the nucleotide arrangement in DNA is antiparallel, so the same definition holds, also, for the bonding paths through the two strands.

- The double helix of DNA is characterised by a minor and major grove. This means that one of the helix is slightly shifted axially relative to the other.

The concept of energy flow through the single strand of the DNA molecule (with the shape of a helix) could be investigated by comparing it with the magnetic field of a solenoid. In this approach, the double helical configuration of DNA could be represented as an equivalent circuit of two parallel, closely spaced bifilar solenoids on a common axis. Now let consider that the cascade energies flow through both strands in opposite directions. In the equivalent circuit of the two solenoids, this will correspond to opposite currents. In this case, the magnetic lines in the internal region of the solenoids will have antiparallel direction, while the external magnetic lines will be closed in proximity of both ends of the solenoids.

Let us call this type of field a "complementary compensated solenoid type". The magnetic lines of this case are schematically illustrated in Figure 7. The two bifilar solenoids that simulate the two strands are shown by green and red colours. Their magnetic field lines, shown as dashed lines, are antiparallel inside the solenoids, while they are connected in proximity at both ends. The energy flows in both solenoids are opposite (noted by black arrows). This solenoid model exhibits some features of a bifilar winding. If attempting to separate both solenoids, they will oppose.

The analysis of magnetic fields with such spatial configuration reveals the following additional features:

(h) The configuration of the associated magnetic field is independent of the secondary (supercoiling) shape of DNA

(i) This type of magnetic field will provide an additional strength of the connections between both strands. It will oppose the separation between the strands because this leads to an increase in the closed path lengths of the magnetic lines

(j) For a small portion of DNA molecule, the complementary compensated solenoidal type of field is axially symmetrical.

The whole mechanism will be characterised by the following features:

(k) the rotated excited states in $(\mathrm{O}+4 \mathrm{C})$ rings will possess the same chirality determined by the direction of the cascade energy flow through the strand to which they are attached

(l) the rotating energy states are phase-synchronized along the DNA strand

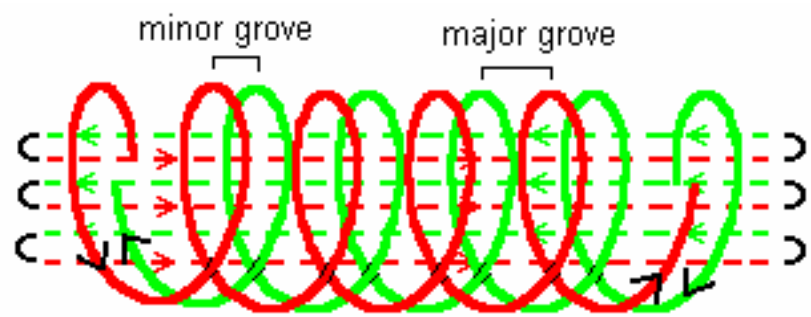

Figure 7: Association of energy flow conditions between DNA strands with complementary compensated bifilar solenoids. (m) the rotating energy states sustain the tendency of unidirectional cascade energy flow through the DNA strand.

Keeping in mind features (a), (b), and (c), the rotating energy states in the ring could be mutually dependant. It is apparent, also, that the mutually dependant features (g), (h), (i) and (j) will lead to a selfsustained mechanism.

These considerations may shed some light on the hydrophobic mechanism existing in the space between the two DNA strands. The two bond angles of the water molecule are illustrated in Figure 8. (discussed in Chapter 9 of BSM-SG) [3].

If a water molecule is placed inside the symmetrical field of the compensated solenoids, the angular positions of the valence electron orbits evidently will be in conflict with the fields of the solenoids. The interaction of the orbiting electrons with these fields may produce expelling forces for this type of molecule. This might explain the hydrophobic environments of the internal region of DNA between the two strands. The hydrophobic environment is quite important for $\mathrm{H}$-bondings between purines and pyrimidines.

The analysis of compensated double solenoid model for the energy flow through DNA leads to the following conclusion:

(A). The DNA double helix molecule could be easily folded into any shape under influence of external factors. The external factors could be various kinds of proteins.

Magnetic field conditions for proteins: It is well known that in appropriate environments, the proteins, which usually possess a complex tertiary structure, preserve their native shape, although the linear DNA molecule, when it is free of bending proteins, does not exhibit this feature. The main reason for the different behaviour of the proteins and the DNA of linear type is the structural differences between them. The protein is a long single strand molecular chain with diversified sequences of amino acids. The protein backbone does not contain low order repeatable structures as does the DNA nucleotide. It may contain, however, higher order repetitions that may form some helices with a small number of turns or other spatial configurations. In this arrangement, the conditions for $\mathrm{H}$-bonding are significantly reduced. All these structural differences indicate that we could not apply the concept of the complementary compensated solenoids for the proteins, as was done for the analysis of DNA.

The different higher order shapes of the proteins might be a result of the diversified sequence of the amino acids. This conclusion is

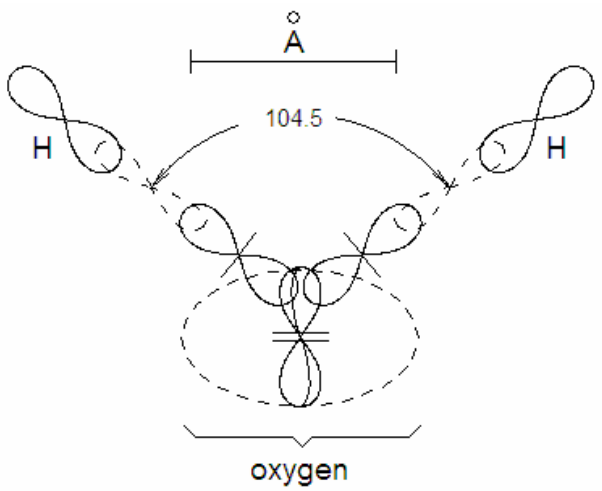

Figure 8: Water molecule using BSM-SG atomic models (only central hadrons of oxygen atom are shown). 
supported by the analysis of the shape of a tRNA molecule illustrated in Figure 9.

The tRNA molecule possesses a single strand of about 77 base pairs but the repeating order of the nucleotides is similar to the DNA molecule. Its single strand arrangement obtains a stable shape of a "cloverleaf". In this arrangement, the unidirectional magnetic field through the single chain of tRNA becomes a type closer to the complementary compensated solenoid, so the principle of shortest magnetic lines is satisfied. The magnetic lines through the strand are connected in the proximity of the open ends and this is the area where the tRNA molecule attaches an appropriate amino acid molecule.

The proteins synthesized according to the DNA code have various sequences of amino acids and exhibit a large diversity in their taxonomy. While the model of the complementary compensated solenoids is not valid for the proteins, they could be analysed by a single solenoid model. If we assume that a unidirectional cascade state transfer is possible through the bonding path of the chain, then a single solenoid model could be used for the helices of the protein. In this case the analysis of secondary and tertiary structures of the proteins leads to the following considerations:

(a) Various types of secondary structures as helices, sheets, beta turns, bulges and so on provide various structures of the magnetic fields.

(a) The magnetic field from the unidirectional cascade state transfer may influence the common positions of the secondary structures

(c) The bond angles between neighbouring atoms are restricted by the strong conditions of finite nuclear structure and restricted angular freedom of the chemical bonds

(d) A discrete type of rotational freedom with some finite deviation is possible between single valence bonds

(e) These considerations and spatial restrictions lead to the conclusion that an overall asymmetry of the magnetic field from the secondary structures (mainly from the helices) may exist. Then, the tertiary structure may try to restore the asymmetry of the magnetic fields arising from the diversified secondary structures by acquiring an

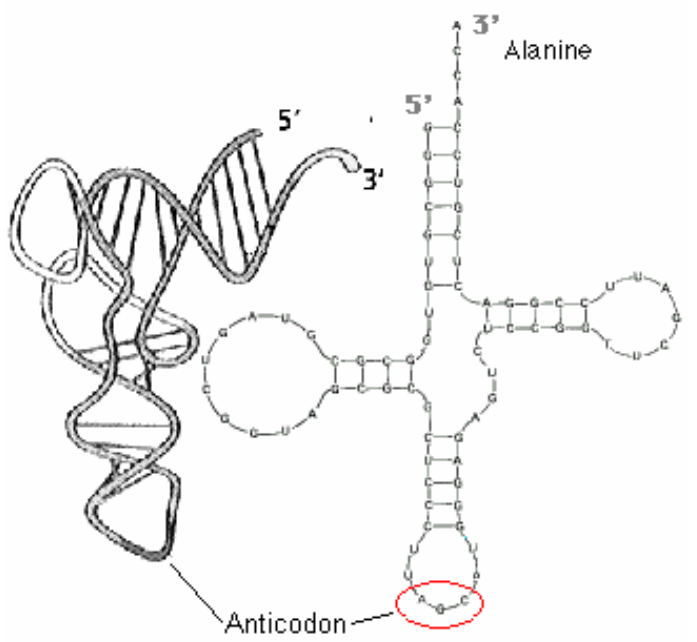

Figure 9: tRNA molecule. (a) - real shape, (b) plane drawing, showing the loops and base pairs (adopted from a public domain). overall symmetry in some higher structural order. Simultaneously, this will be accompanied by a tendency to shortest magnetic lines.

(f) The cascade energy transfer through the bonding path of the protein chain might be accompanied with some ion current, whose energy could also be involved in the total energy balance.

The taxonomy of $3 \mathrm{D}$ protein structures is well described in a text book [5]. The stiffness of the secondary order structure is larger than the stiffness of the tertiary one. This matches the strength of the mechanism involved in the restoration of overall field symmetry. As a result, every protein may have a sustainable native shape when placed in appropriate environments (temperature, $\mathrm{pH}, \mathrm{ATP}$ ).

Our considerations helps to explain one of the problems in molecular biology, known as Levinthal's paradox: "Why do the proteins fold reliably and quickly back to their native state despite the astronomical number of the possible quantum states according to Quantum mechanics?".

Now let us consider how the protein may react if the condition of shortest magnetic lines is temporarily disturbed by some external factor; for example, by addition of Adenosine triphosphate (ATP). The ATP carries stored energy in its rings. When a chemical reaction takes place, the stored energy might be induced in some rings in the protein under consideration. This may change the conditions for which the principle of shortest magnetic lines and compensated field is satisfied. Then the reaction leading to restoration of the energy balance (involving the magnetic energy) could involve some kind of change of shape and motion of the protein. This may eventually explain the protein motility in appropriate environments (temperature, $\mathrm{pH}$, ATP concentration) that are simultaneously accompanied by a temporary change of its higher order shape.

Environmental considerations for preservation of the native shape of biomolecules: Many proteins have a complex 3D structure with secondary helix and tertiary structure. They exhibit amazing tendency to preserve their $3 \mathrm{D}$ shape in appropriate environment conditions in which the temperature is one of most important factors. The electronic bonds and hydrogen bonds allow some freedom of the 3D structure of biomolecules. It is known from the Quantum mechanics that the excitation of a particular vibrational-rotational band of the molecular spectra is temperature dependant. Translated into BSM-SG theory, this dependence means:

- Self-adjustment of the quantum energy level corresponding to appropriate subharmonic number from the available set of the quantum orbits [5].

- A proper quantum number (according to BSM-SG models)

Consequently, the complex $3 \mathrm{D}$ shape of the biomolecules is dependent on two main features:

(a) An appropriate subharmonic and quantum number for every bonding electron (see page II-0 of Atlas ANS ) $[2,3,6]$.

(b) The same subharmonic and quantum number of the similar interatomic bonds along the molecular chain

Feature (a) provides a requirement for the absolute temperature range for the preservation of the native state of a long chain molecule, while feature (b) puts a strict requirement for temperature uniformity along the molecular chain. This, of cause, is not the only factor. Additional factors are the interactions between the highly oriented atomic ring structures discussed in previous paragraphs and the 
conditions for H-bondings. Other environmental conditions that are also important, for example, are such as the $\mathrm{pH}$ factor and ATP concentration. The first one may assure appropriate ion current conditions (according to consideration ( $\mathrm{f}$ ) in section 5.2, while the second one - the necessary energy.

Magnetic fields involved in the higher order structures of DNA: The DNA molecules of some single cell organisms are usually of a circular type with supercoiling. The DNA of eukaryotes, however, is of a linear type with many more base pairs and complicated secondary and third order structures. According to the principle of the shorter magnetic lines and compensated magnetic field, circular DNA should be more resistant because the magnetic line paths are enclosed inside the structure. This condition might be partly disturbed during the transcription only. Linear DNA, however, possesses some additional properties that may facilitate the process of intermolecular DNA communications, according to a hypothesis presented below.

The length to diameter ratio of DNA is very large. It is extremely large especially for eukariotic DNA. For human chromosomes, for example, the average ratio is of the order of 107 . To be held in the very small space of a cell nucleus, these long molecules are coiled a higher order structure known as supercoiling. The mechanisms involved in the formation and stability of this structure have not been completely understood so far. The following analysis sheds some light on the possible mechanisms.

Let us first present briefly some of the experimental observations about the supercoiling features of a long DNA molecule. In the article "DNA-Inspired Electrostatics" in Physics Today by Gelbart et al. [7], the authors provide a summary about one important feature of DNA. They say: "Under physical conditions (a 0.1 molar solution of $\mathrm{NaCl}$ ), a DNA molecule takes on the form of a disordered coil with a radius of gyration of several micrometers; if any lengths of the molecule come within $1 \mathrm{~nm}$ of the other, they are strongly repelled. But, under different conditions - as in a highly diluted aqueous solution that also contains a small concentration of polyvalent cations - the same DNA molecule condenses into a tightly packed, circumferentially wound torus."

The DNA molecule is usually negatively charged, so the repulsion between the various parts of the long chain molecule about $1 \mathrm{~nm}$ apart is understandable. However, why does the DNA fold into such a packed toroidal structure in the presence of appropriate polyvalent cations? This effect has a reasonable physical explanation within BSMSG theory if we analyse the magnetic field conditions at the CL (Cosmic Lattice) node level [2].

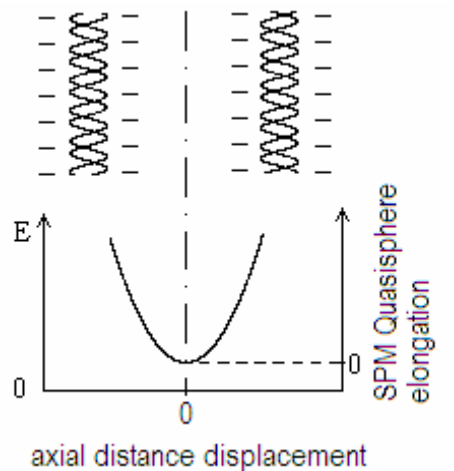

Figure 10: Electric field and SPM Quasisphere elongation between two parallel parts of DNA.
Figure 10 illustrates the electric field intensity between two parallel parts of DNA. The vertical axis scale on the left shows the electric field intensity, while the right vertical axis shows the elongation of the SPM quasispheres according to BSM-SG (see \$2.9.4 Chapter 2) [2].

One specific feature is apparent from the drawing in Figure 10. The elongation of SPM quasispheres becomes zero in the middle between the parallel strands. This is possible only because of the strong spatial orientation of the electric Quasispheres (EQ) (contributed to the E-field) along the parallel parts of DNA. Consequently, the elongation of all SPM quasispheres in the plane passing through the parallel DNA axes is reduced to zero. Then these SPM quasispheres become MQ type (Magnetic Quasispheres). At the same time the SPM vector of these MQs might be synchronized, because the interacting EQs from the parallel long chains could be easily synchronized. Then the MQs obtained in such way provide excellent conditions for a permanent magnetic field. To make its direction permanent, however, it needs some stabilisation, which can be obtained by some interaction with an external current. This current may be provided by heavy and polyvalent ions, while the masses of $\mathrm{Na}$ and $\mathrm{Cl}$ are not much different and their charge is not large enough. The flow of the positive and negative ions are expected to have different paths. This could happen if they have different masses because they will have different centripetal accelerations in a helical trajectory. The supercoiling shape of long DNA will provide a necessary condition for this trajectory. At the same time, the condition of shorter magnetic lines will keep the supercoiled DNA in a compact configuration, while the negative charge of DNA strands will keep the appropriate distance between the different parallel parts of the molecule.

The explanation of the DNA supercoiling in eukariotic cells in a natural cell environment requires some additional considerations. Human DNA, for example, is supercoiled around a protein called histon octamer. The shape of the higher order structures of DNA is a nucleosome formation, a Chromatin and a Chromosome are presently well known. They are illustrated by Figure 11.

Conclusion (A) in $\$ 5.1$ provides an explanation of why the DNA molecule is easily folded as a secondary helix around the histone octamer. The latter, evidently, provides a higher order helical shape condition. According to the considerations in $\$ 5.1$ and $\$ 5.2$, the histones may also have features of energy flow. If we examine the atomic structures of

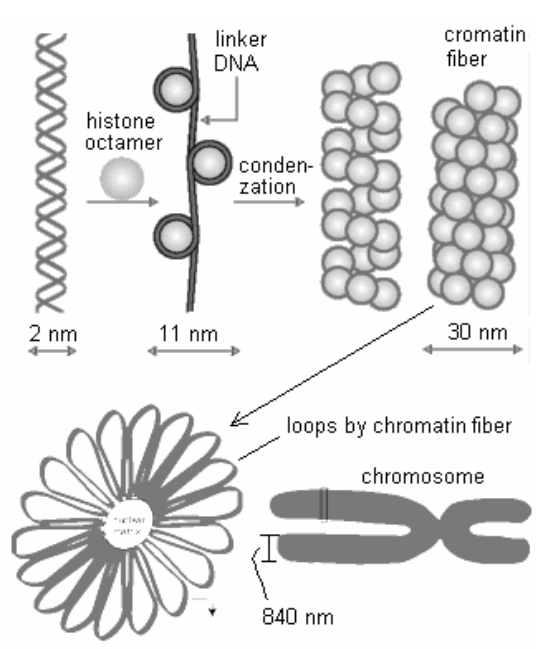

Figure 11: Higher order structures of DNA in Chromatin and Chromosome (adopted from a public domain). 
the histones (using the Protein data Bank) we will find that two types of atomic rings are involved: (6C) and $(\mathrm{N}+4 \mathrm{C})$. A possible energy flow through the spatially arranged rings may contribute to the bending and coiling of the DNA around the histone octamer.

Chromatin, known as a chromatin fibre (with diameter of $30 \mathrm{~nm}$ ) is additionally coiled into a daisy-like shape, called a chromosome miniband. The chromosome miniband, shown in the lower left side of Figure 11 contains 18 loops of daisy-like shape. A stack of daisy-like minibands arranged in a super helix with a diameter of about $840 \mathrm{~nm}$ forms a chromosome.

The discussed effect of magnetic field creation with a sustainable ion current may be part of the complex mechanism sustaining the supercoiled structure of the human chromosome. The balance between the magnetic field, the ion current, and the electrochemical potential will depend on the internal cell environments as in the case of proteins. Simultaneously, the principle of shorter magnetic lines and compensated magnetic field should also play some role. When one of the two ends of the chromosome is attached to the cell, the ion current and magnetic field will be stabilized, so the supercoiled shape will be stable. If the chromosome, however, is not attached for some reason, a small change in the ion current will cause a reaction of the magnetic field. This may invoke a shape change and a motility of the chromosome.

\section{Hypothesis for the role of DNA in the cell cycle synchronization}

General considerations: Cell cycle synchronization is an important factor for normal organ formation in the growing multicellular organism. One simple example demonstrating this importance is the eye formation. Some non-synchronized cell division in an early phase of eye formation may lead to significant optical defects. Then, how do the enormous number of individual cells know the exact instant to enter into mitosis? If only individual clocks mechanisms in the cells are involved, they could lose synchronization after a number of cycles. Synchronization by chemical messengers through the bulk of an enormous number of cells is not a satisfactory explanation.

It is reasonable to expect that DNA might be involved in cell cycle synchronization. The analysis of DNA at the atomic level within BSMSG theory led to the hypothesis of electromagnetic intercommunication of DNA molecules located in various cells of the same type. The proposed hypothesis suggests a physical mechanism that might be involved in the synchronization process.

Let us use the concept of complementary compensated solenoids as in $\$ 5.1$, illustrated by Figure 7 but regarding both complementary parts of the helical system as separate helices. In this case, they could be considered as two solenoids serially connected in a closed circuit. Then the direction of cascade energy transfer through both strands of DNA (corresponding to the two separated solenoids) could be clockwise or counter clockwise. This will define two different directions of the magnetic lines. Let us suppose that the resulting magnetic field is involved also in some interaction processes in the cell. This means that the direction of the cascade energy flow relative to the helical direction will be kept stable. Let us denote the direction of a stable energy cascade through one strand as $+z$. This direction, regarded as axis $+z$, is not a straight line but a helix geometrically centred on one of the DNA strands. The stable energy flow through this strand will obviously influence the direction of the rotational energy states in the attached $(\mathrm{O}+4 \mathrm{C})$ rings. Thus we may assume that they all have a clockwise direction coinciding with the rotating direction of the running energy cascade through the helical DNA strand along the $+z$ axis. In other words, all stored energies in the $(\mathrm{O}+4 \mathrm{C})$ rings have the same chirality. We note that the definition of chirality is referred to the direction of the cascade energy flow.

Let us now discuss the energy storage capability of the purines and pyrimidines involved in DNA. The pyrimidines Cytozyn (C) and Thymin $(\mathrm{T})$ have single rings $(2 \mathrm{~N}+3 \mathrm{C})$ with electronic bond connections to the DNA strands. The purines Adenine (A) and Guanine $(\mathrm{G})$ both have two attached ring structures $(2 \mathrm{~N}+3 \mathrm{C})$ and $(2 \mathrm{~N}+4 \mathrm{C})$ that are also connected to the DNA strands. Because the single rings of $\mathrm{C}$ and $\mathrm{T}$ are the same as the attached rings of A and G, we may assume that they carry the same amount of stored energy.Purines and pyrimidines have stronger bond connections to their own strand than between themselves as base pairs. The chirality of their stored energies could also be influenced directly by the chirality of the energies in the $(\mathrm{O}+4 \mathrm{C})$ rings from the strands. Using the same logic, the $(2 \mathrm{~N}+3 \mathrm{C})$ rings should acquire the appropriate chirality from the strands to which they are strongly connected. The $(2 \mathrm{~N}+4 \mathrm{C})$ rings attached to them, however, will acquire a complementary opposite chirality.

Now let us consider the normal situation, when both strands of DNA are closely spaced in the shape of a double helix. If we align the DNA along a new defined axis $+Z$, the rotating energy states in the rings connected to one selected strand, say the first one, will have a clockwise rotation, while from the second one - a counter clockwise. Consequently, when introducing a common direction axis $(+\mathrm{Z})$ for both strands, the rotating energies from $(\mathrm{O}+4 \mathrm{C})$ connected to the two strands of DNA will look as they have different chirality. (In further analysis, the common $+\mathrm{Z}$ axis will be assumed for both strands).

Table 1 provides the chirality of the stored energies for all types of rings in the DNA molecule resulting from the analysis above. The stored energy chirality is referred to a common axis $\mathrm{Z}+$ of the DNA molecule.

Table 1 - Energy and chirality states.

strand A strand B Energy states for

$\mathrm{ES} / \mid \backslash \mathrm{ES} \backslash /(\mathrm{O}+4 \mathrm{C})$ ring

$$
\begin{aligned}
& (\mathrm{E} 1 / \mid \backslash)(\mathrm{E} 2 \backslash / /)------(\mathrm{E} 1 \backslash /) \mathrm{A}---\mathrm{T} \text { and } \mathrm{G}---\mathrm{C} \\
& (\mathrm{E} 1 / \mid \backslash)-------(\mathrm{E} 2 / \mid \backslash)(\mathrm{E} 1 \backslash /) \mathrm{T} \text {---A and C---G }
\end{aligned}
$$

\section{Notations:}

$/ \backslash$ and $\ /$ - two states of chirality

ES - a rotating energy state in $(\mathrm{O}+4 \mathrm{C})$ ring

E1 - a rotating energy state in $(2 \mathrm{~N}+4 \mathrm{C})$ ring

E2 - a rotating energy state in $(2 \mathrm{~N}+3 \mathrm{C})$ ring

----- a connection by a hydrogen bond $(\mathrm{E} 1 / \mid \mathrm{I})(\mathrm{E} 2 \backslash / /)$ - energy states with complementary chirality valid for attached rings

Let us examine how the stored energies in the rings of various types could be influenced by some change in the cascade sequence. The energies of the $(\mathrm{O}+4 \mathrm{C})$ rings can be strongly affected by a change in the energy cascade flow through the strand because the strand bonding path passes through the $\mathrm{C}=\mathrm{C}$ bond of every ring. The stored energies in single $(2 \mathrm{~N}+3 \mathrm{C})$ rings could be affected by the change in the cascade type energy flow through $(\mathrm{O}+4 \mathrm{C})$ rings. The stored energies in the attached rings $(2 \mathrm{~N}+3 \mathrm{C})$ and $(2 \mathrm{~N}+4 \mathrm{C})$, however, is of a complementary type and is more resistant to the energy flow changes mentioned above.

Summarizing, we may conclude: (1) The stored energy sequence 
Citation: Sargoytchev SS (2017) A New Method for Analysis of Biomolecules Using the BSM-SG Atomic Models. J Biom Biostat 8: 339. doi:10.4172/2155-6180.1000339

Page 9 of 16

\begin{tabular}{|c|c|c|c|c|c|c|c|}
\hline $\begin{array}{l}\text { UUU } \\
\text { UUC }\end{array}$ & Phenvlalanine & \multirow[t]{3}{*}{$\begin{array}{l}\text { UCU UCC } \\
\text { UCA UCG }\end{array}$} & \multirow[t]{3}{*}{ Senile } & $\begin{array}{l}\text { UAU } \\
\text { UAC }\end{array}$ & Tyrosine & $\begin{array}{l}\text { UGU } \\
\text { UGC }\end{array}$ & Cvsteine \\
\hline \multirow{2}{*}{$\begin{array}{l}\text { UUA } \\
\text { UUG }\end{array}$} & \multirow[t]{2}{*}{ Leucine } & & & \multirow{2}{*}{$\begin{array}{l}\text { UAA } \\
\text { UAG }\end{array}$} & Stop & UGA & Stop \\
\hline & & & & & Stop & UGG & Tryptophan \\
\hline \multirow[t]{2}{*}{$\begin{array}{l}\text { CUU CUC } \\
\text { CUA CUG }\end{array}$} & \multirow[t]{2}{*}{ Leucine } & \multirow[t]{2}{*}{$\begin{array}{l}\text { CCU CCC } \\
\text { CCA CCG }\end{array}$} & \multirow[t]{2}{*}{ Proline } & $\begin{array}{l}\text { CAU } \\
\text { CAC }\end{array}$ & Histidine & CGU CGC & \multirow[t]{2}{*}{ Arginine } \\
\hline & & & & $\begin{array}{l}\text { CAA } \\
\text { CAG }\end{array}$ & Glutamine & CGA CGG & \\
\hline $\begin{array}{l}\text { AUU } \\
\text { AUC }\end{array}$ & Isoleucine & \multirow[t]{2}{*}{$\begin{array}{l}\text { ACU ACC } \\
\text { ACAACG }\end{array}$} & \multirow[t]{2}{*}{ Threonine } & $\begin{array}{l}\text { AAU } \\
\text { AAC }\end{array}$ & Asparagine & $\begin{array}{l}\text { AGU } \\
\text { AGC }\end{array}$ & Senile \\
\hline $\begin{array}{l}\text { AUA } \\
\text { AUG }\end{array}$ & Stan; Methionine & & & $\begin{array}{l}\text { AAA } \\
\text { AAG }\end{array}$ & Lysine & $\begin{array}{l}\text { AGA } \\
\text { AGG }\end{array}$ & Arginine \\
\hline \multirow[t]{2}{*}{$\begin{array}{l}\text { GUU GUC } \\
\text { GUA GUG }\end{array}$} & \multirow[t]{2}{*}{ Valine } & \multirow[t]{2}{*}{$\begin{array}{l}\text { GCU GCC } \\
\text { GCA GCG }\end{array}$} & \multirow[t]{2}{*}{ Alanine } & $\begin{array}{l}\text { GAU } \\
\text { GAC }\end{array}$ & $\begin{array}{l}\text { Aspartic } \\
\text { acid }\end{array}$ & \multirow[t]{2}{*}{$\begin{array}{l}\text { GGU GGC } \\
\text { GGA GGG }\end{array}$} & \multirow[t]{2}{*}{ Glycine } \\
\hline & & & & $\begin{array}{l}\text { GAA } \\
\text { GAG }\end{array}$ & $\begin{array}{l}\text { Glutamic } \\
\text { acid }\end{array}$ & & \\
\hline
\end{tabular}

Table 1: Codons involved in RNA.

in $(\mathrm{O}+4 \mathrm{C})$ rings (with unit value of ES) is strongly influenced by a change in the cascade energy flow through the DNA strands

(2) The complementary energies $(\mathrm{E} 1 / \mid \backslash)(\mathrm{E} 2 \mid / /)$ in the attached rings are more stable than the energy E1 in the single ring.

(3) A change of energy sequence in $(\mathrm{O}+4 \mathrm{C})$ rings could influence the stored energies more strongly in the single $(2 \mathrm{~N}+3 \mathrm{C})$ rings than in the $(2 \mathrm{~N}+4 \mathrm{C})$ rings.

(4) The energies E1 and E2 connected by $\mathrm{H}$ bonds A--T and G--C have always the same chirality.

Now let us assume that the DNA strands are opened at one end only. In this case the paths of the magnetic lines is increased at this end. It is known from physics that if an alternating field component appears in this case, some energy will be radiated as EM waves. At this point, however, we must consider some specific features of the double helix structure of DNA: The $(\mathrm{O}+4 \mathrm{C})$ rings, arranged in a helix, contain equally spaced gaps. Keeping in mind the enormous number of these rings, the cascade energy transfer will have a very large unidirectional component but a very small alternating component for one clock (step). Then it is reasonable to assume that the generated magnetic field will have the same unidirectional feature as the field from the energy cascade through the strands. This means that every emitted pulse will have an energy of $2 \mathrm{ES}$.

When we consider the energies of single rings $(\mathrm{N} 2+\mathrm{C} 4)$, however, the emission conditions are different. They do not form an uninterrupted sequence along the chain as do the $(\mathrm{O}+4 \mathrm{C})$ rings, so the sequence of the released E1 energies cannot possess their own permanent component. This might affect the preferred direction of the generated magnetic field. The helicity of DNA and higher order helicity of chromatin and chromosome with some complementary ion currents may also provide conditions of energy release from $(\mathrm{O}+4 \mathrm{C})$ rings in the same direction for both strands. It is reasonable to suppose that the radiation direction could follow the direction of emptying the energies of $(\mathrm{O}+4 \mathrm{C})$ rings. Let us assume that this direction corresponds to the introduced $+Z$ axes. Figure 12 illustrates the sequence of emptying the stored energies in $(\mathrm{O}+4 \mathrm{C})$ rings (with individual energy $\mathrm{ES})$ and $(2 \mathrm{~N}+4 \mathrm{C})$ rings (with energy E1). The energy status and the direction of energy emission are shown for two consecutive clocks (steps) (i) and $(\mathrm{i}+1)$. The energies stored in the two attached rings $(2 \mathrm{~N}+4 \mathrm{C})$ and $(2 \mathrm{~N}$ $+3 \mathrm{C}$ ) are not shown in the figure, but they are always complementary to the $(\mathrm{O}+4 \mathrm{C})$ rings.

The chirality of all ES energies relative to the direction of the
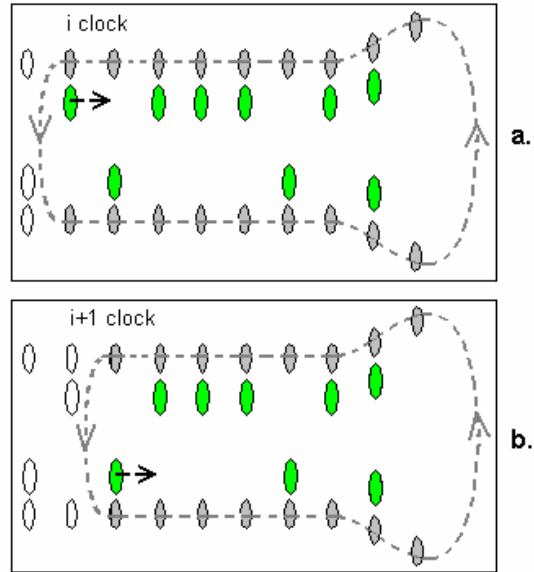

0 Ring with $\mathbf{E}_{\mathbf{s}} \quad-\rightarrow$ Emission direction of $\mathbf{E}_{\mathbf{s}}$
0 Ring with $\mathbf{E}_{\mathbf{1}} \quad-->$ Emission direction of $\mathbf{E}_{\mathbf{1}}$
0 Empty ring

Figure 12: Energy status and direction of energy transfer for two consecutive clocks. The radiation direction is along $+Z$ axis.

permanent magnetic field (defined by the cascade energy transfer through the bonding path) indicated by arrows in a dashed line is one and a same. When emitted by the mutual $+\mathrm{Z}$ direction, the corresponding ES energies contribute to a single 2ES pulse. For E1 energies, however, the emission conditions are different because they do not have a stable unidirectional sequence. Then, considering the energy emptying direction $+Z$, the chirality from $E 1$ belonging to the different strands is different (clockwise or counterclockwise relative to the $+\mathrm{Z}$ axis). Let us consider:

(a) The $\mathrm{E} 1$ energies with clockwise chirality relative to the $+\mathrm{Z}$ axis provide a photon sequence with a clockwise phase difference between the photons.

(b) The E1 energies with a counter clockwise chirality relative to the $+Z$ axis provide a photon sequence with a counter clockwise phase difference between the photons.

(c) The emitted photons of E1 energies from the same strand are entangled with the preserved phase difference. The phase advance in one strand (with $a+Z$ orientation of its cascade energy) will have a 
clockwise phase sequence, while from the other strand it will be counter clockwise.

(d) Either sequence of entangled photons contains embedded information about the A--T and G--C sequence of DNA referred to one strand.

(e) The energy of the entangled photon sequence is less dispersive in the conditions of the intercellular medium.

(f) The probability of absorption of the entangled photons is much higher if they encounter a similar spatially arranged atomic ring.

(g) If the process of DNA energy release is invoked, not by internal factor but by externally induced synchronization, it might have some phase delay due to a missing part of the initial synchronization sequence.

Photon entanglement is observed in lasers. At first, two entangled photons were observed. Observation of three entangled photons was published by the Zelinger group. The same group later reported observation of four entangled photons. Observations provided by entangled photons show that they contravene a dispersion when moving through inhomogenious media. This may provide an explanation for feature (e)

It is reasonable to consider that conditions of uniform temperature and appropriate spatial arrangement of the ring structures in DNA are necessary requirements for multiple photon entanglement in the mechanism described above. It is known from single-mode narrowline lasers that their coherence time is quite long. Let us assume, for example, that the coherence time in the DNA emitting process is of the order of

$1 \times 10^{-10}(\mathrm{~s})$. If the average lifetime for a single bond is about $1 \times$ $10^{-12}(\mathrm{~s})$, then the time between two ES clock pulses is $6 \times 10^{-12}(\mathrm{~s})$ ( $\mathrm{six}$ bonds). In this case, the entangled sequence will include a binary code corresponding to $600 \mathrm{ES}$ clock pulses. This binary code corresponds to $600 \mathrm{bp}$ (base pairs) and involves 200 codons.

C-value paradox: Now let us consider a particular problem related to DNA research, known as the $\mathrm{C}$-value paradox. A substantial fraction of the genomes of many eukariotes, for example, is comprised of repetitive DNA in which short sequences are repeated in tandem in small to large arrays. This unexplained so far problem is known as a C-value paradox. Repetitive tandem sequences, known as "satellite" DNA's, are classified into three major groups;

-Satellites with repetition lengths from one to several thousands base pairs.

- Minisatellites - repetitive arrays of 9 to $100 \mathrm{bp}$, but usually $15 \mathrm{bp}$, generally involved in mean arrays lengths of 0.5 to $30 \mathrm{~kb}$.

- Microsatellites - repetitive arrays of 2 to $6 \mathrm{bp}$ found in vertebrate, insect and plant genomes.

The code of the repetitive sequence does not encode amino acids. The percentage of this non-informative DNA increases significantly with organism complexity but depends also on other factors. Among them are the environmental conditions of the species.

From the point of view of our proposed hypothesis, the C-value paradox has quite logical explanation:

(h) The repetitive sequences in DNA code might serve as a synchronization code that may increase the reliability of the DNA intercellular communication.
Many diversified features of DNA code redundancy in various organisms and species could find a logical explanation from the point of view of feature (h).

For example, the repetitive sequence in DNA from Drosophilia shown below corresponds to the following binary codes embedded in two entangled photons related to both strands:

(AATAT)n, where $\mathrm{n}$ - is the number of repetitions:

- from DNA strand 1: (00101)n

- from DNA strand 2: (11010)n

If the entangled photon emitted from strand 1 contains CW (clock-wise) circular phase sequence of the code, the entangled photon emitted from strand 2 will contain CCW (counter clock-wise) circular phase sequence of the complementary code.

It is evident that the entangled photons carry embedded information that is dependent on both: the amino acid code and the redundancy code. This dependence may eventually play a role in the immunological response to transplanted tissues or organs. The redundancy code greatly increases the probability of successful synchronization. Then it is reasonable to expect a large abundance of repeatable code near the end side of the linear DNA in eukariotic cells. Such redundancy has been observed.

Time sequence in the energy read-out process of DNA and its possible relation to cell cycle synchronization: Let us consider some initial state of the DNA molecule when all rings contain their normal energies ES, E1 and E2. At some particular moment invoked by internal or external triggering, the stored energies begin to clock out with the synchronization sequence ES and two entangled photon sequences. Once this process is started, it will be self-sustained until all ES energies states are read out. We may call this a read-out process of DNA. The time duration $\left(\mathrm{t}_{\text {total }}\right)$ for DNA read-out should be $\mathrm{t}_{\text {total }} \approx 6 \mathrm{t}_{\mathrm{ar}}$ DNA length/0.34 $\mathrm{nm}$.

Where: $t_{\text {av }}$ - is the average lifetime of excited states in the bonding path of a DNA strand; $0.34 \mathrm{~nm}$ is the distance between the rings of neighbouring base pairs.

The total read-out time is very short. For DNA with length of $1 \mathrm{~m}$ and $\mathrm{t}_{\mathrm{av}} \sim 1 \times 10^{-12}(\mathrm{~s})$ the read-out time should be only $1.8 \mathrm{~ms}$.

After the DNA is read out, all ES energies are emitted. Only the E1 energies of $\mathrm{T}$ and $\mathrm{C}$ are emitted, but the complementary state energies $\mathrm{E} 1$ and $\mathrm{E} 2$ of $\mathrm{A}$ and $\mathrm{G}$ are preserved due to the attached rings. This invokes some type of asymmetry between connected purines and pyrimidines by H-bonds. These conditions may help in the separation of the DNA strands to initiate the replication process.

Now let us analyse the possible involvement of the DNA readout process in cell cycle synchronization in eukaryotes. We will not discuss here the complex processes of cell cycle regulation in which the proteins are involved, but only the conditions of successful triggering and read-out of DNA. We may assume only that every individual cell possesses some kind of triggering mechanism. Such a mechanism is necessary but not a sufficient condition to initiate a read-out process. The DNA read-out can be successful only if conditions for emission of EM energy exist. Consequently, it will depend on the following states of the DNA molecule:

(a) The two strands of one end of DNA must be separated (or unbalanced by a promoter) for the EM quanta (photons) to be emitted, 
(b) The two strands not counting the end conditions mentioned in (a) must be completely symmetrical for the read-out process to be self-sustainable.

Requirements (a) and (b) exclude the mRNA attachment to DNA or any other regulatory protein, so the transcription process and the regulatory mechanisms should be completed. Then, for the synchronized read-out to become an avalanche process, the expected optimal conditions should exist during phase S (start of DNA replication).

Now let assume that the internal triggering mechanism provides trigger pulses with a period much shorter than the cell cycle. These pulses will not provide successful triggering until conditions (a) and (b) are satisfied. However, once they are satisfied, the DNA read-out will be successful. Then the entangled photons emitted with a large common energy and encoded sequence possesses an increased probability of activating a similar read-out process in the DNA of neighbouring cells. The extremely fast read-out process could lead to a similar read-out process in many DNA molecules. In this case, the emitted entangled photons may additionally interfere and contribute to the avalanche process of synchronization. The avalanche process, however, will be supported only by DNA molecules that are not significantly different. In the case of tissue and organ transplantation, the avalanche process will not work between the DNA from different species whose synchronization sequences are too different.

Environment considerations for the efficiency of the avalanche process: The phase accuracy of the cell cycle synchronization evidently depends on the individual cell cycle phases and environmental conditions.

Phase accuracy dependence of the cell cycle period: If we consider the triggering of the synchronization from internal cell mechanism in an appropriate phase of the cell cycle, the period between two consecutive synchronization bursts will depend on the time lag between the local clock triggering events. Evidently, the period of these events should be much smaller than the cell cycle period.

Let us assume that the individual cell cycle regulation mechanisms have a Gaussian distribution of the cell cycle period for many cells. Then the optimum conditions for an avalanche read-out should be expected at some moment that is close to but not beyond the maximum of the Gaussian curve. Once it is initiated, the Gaussian curve will be decreased for all cells, so the cells not initially activated by the synchronization pulses will not contribute. In such a case the cells in which the transcription and regulation processes are not yet completed will be excluded from the process. Their mitosis will not be in phase with the synchronized cells.

Additionally we may not expect that all the synchronized cells will be activated at the very beginning of the read-out of the first activated cells. The repeatable sequences of codons may provide conditions for a larger number of entangled photons. Such photons also posses an increased probability for selective absorption and activation of similar repetitions from DNA in other cells.

Consequently, the tandemly repetitive sequences of DNA provide a higher probability for triggering of an avalanche process of synchronization.

Physical factors of cell environment: The most important environmental factor for successful EM synchronization is the temperature. It is reasonable to consider that the cascade energy transfer through the DNA strands is phase synchronized by the energy rotation cycle in the $(\mathrm{O}+4 \mathrm{C})$ rings. The duration of this cycle is:

$$
\mathrm{T}_{\mathrm{R}}=\mathrm{t}_{0}+5 \mathrm{t}_{\mathrm{c}}
$$

Where: $t_{0}$ and $t_{c}$ are the lifetimes of the excited states in oxygen and carbon respectively.

The period $T_{S}$ of the synchronization pulses is defined by the sum of the consecutive lifetimes through the bonding path of the strand, shown in Figure 6.

$$
\mathrm{T}_{\mathrm{s}}=\mathrm{t}_{\mathrm{c}}+5 \mathrm{t}_{\mathrm{av}}
$$

Where: $t_{a v}$ is the averaged lifetime value of the bonding electrons involved in the bonding path of the strand.

Then the condition for phase synchronization is expressed by the relation:

$$
\mathrm{T}_{\mathrm{s}}-\mathrm{T}_{\mathrm{R}}=\mathrm{t}_{0}-4 \mathrm{t}_{\mathrm{c}}-5 \mathrm{t}_{\mathrm{av}}=\text { const }
$$

Expression (5) is a constant for all involved DNA molecules if the same energy levels of the excited states are involved. This is a quantum mechanical condition that will depend only on the temperature. The successful generation of entangled photons, however, is additionally dependent on the correct spatial arrangement of the $(2 \mathrm{~N}+4 \mathrm{C})$ rings. If the DNA molecule is sharply bent or is in proximity to a protein involving magnetic field asymmetry, then the condition of multiple photon entanglement will be disturbed. This will affect the efficiency of the synchronization process. Formations of Cruciforms, for example, may affect the emitted sequence. Z-type DNA inclusions, and any external influences causing a helical non-uniformity of Beta type DNA may also block the successful read-out process.

Synchronization is possible in a limited temperature range and adequate temperature uniformity along the DNA chain Synchronization efficiency is dependent on the bending conditions of DNA and the spatial and helical uniformity along its chain. External factors causing any modification of the spatial parameters of the DNA double helix may inhibit the readout process.

\section{Features and experimental results supporting the hypothesis of DNA involvement in the cell cycle synchronization}

The proposed hypothesis is supported by a large number of established features of DNA molecules and experimental observations.

Absorption properties of DNA: DNA absorbs ultraviolet light in the range of 240 to $280 \mathrm{~nm}$ with a maximum at about $260 \mathrm{~nm}$. A temperature increase destabilizes the double helix. Experimental observations lead to the conclusion that the thermal stability of DNA depends not only on the hydrogen bonding, but also on base stacking (Saenger) [8]. This is in agreement with the suggested hypothesis of magnetic field stabilisation generated by the cascade energy flow through the bonding paths of DNA strands.

The mechanism of energy storage and release, according to suggested hypothesis, is in agreement with the observations reported. In this article "Intrinsic Fluorescence from DNA can Be Enhanced by Metallic Particles" [9]. In their experiments they use silver particles of size about $4 \mathrm{~nm}$ on the surface of two quartz plates separated by $1-.5$ um. The DNA placed in this gap is then excited at $287 \mathrm{~nm}$ with pulse sequence from a dye laser with a pulse width of $100 \mathrm{ps}$. The intrinsic emission following this excitation is in the range from about 330 to $350 \mathrm{~nm}$ and is increased about 80 times in the area around the metal particles. The fluorescence is thought to be from adenine and guanine. The authors explain the effect as a decreased lifetime in relation to the 
SERS effect (Surface-enhanced Ramman spectroscopy). Nevertheless, they also acknowledge the existence of another less understood effect. The results for time dependent intensity decay are shown in Figure 13 (corresponding to Figure 5 of their publication).

The explanation of the effect from a BSM-SG theory point of view and the suggested hypothesis is the following. The laser pulse charges the energy storage rings of DNA. The silver metal islands introduce non-uniform field conditions in proximity to the DNA. In this case, the mechanism that may keep the stored energy in the rings is disturbed. Then, in the case when DNA is close to the metal islands, a spontaneous read-out process may occur sooner than a normal one. This will appear in a shorter time interval between the excitation pulse and the fluorescence emission in comparison to the case when DNA is not in proximity to such particles. This corresponds to the observed decreased lifetime. It is interesting to note that the fluorescence lifetime is still much longer than the lifetime in a single electronic bond. This confirms the idea that the stored energies in the rings may have a number of rotational cycles.

Increased binding ability of some simple organic molecules: The proteins that bind to DNA are usually very large molecules containing thousands of atoms. But some comparatively simple organic molecules also have an increased ability for binding. When examining such molecules we see that they possess attached atomic rings. As was mentioned earlier, the energy in such rings is more stable. This gives them additional ability to interact with the DNA molecule by magnetic field interactions. Such molecules for example are:

Trimethylpsoralen: 3 attached rings

Ethidium Bromide: 3 attached rings +1 single

Chloroquine: 2 attached rings.

Effects of the salt concentration in helical winding and DNA supercoiling: As the concentration of a monovalent cation $\left(\mathrm{Na}^{+}\right)$or divalent cation $\left(\mathrm{Mg}^{2+}\right)$ increases to high levels, the DNA double helix winding becomes less tight. According to the suggested hypothesis, the ion concentration influences the ion currents involved in the stabilisation of the magnetic field of DNA, responsible for its supercoiling shape.

Role of intermolecular triplexes in genetic recombination: Triplex DNA-forming regions have been identified near the sites involved in genetic recombinations. They usually have a mirror symmetry repetition of base pairs (as AGGAG). Kohwi and Panchenko

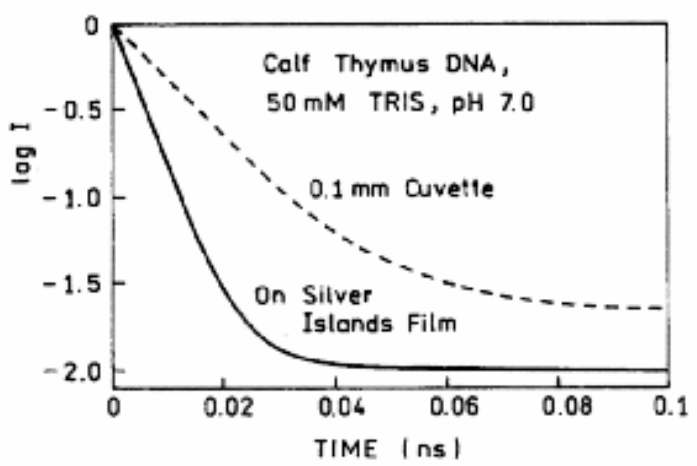

Figure 13: Time-dependent intensity decay of DNA without metal (-) and between silver island film (Courtesy of Lakowicz et al.) [9].
[10] found that the formation of intramolecular triplex structures in DNA in vivo can induce genetic recombination between two direct repetitions flanking the triplex-formed sequence.

According to the proposed hypothesis, triplex-formation in DNA disturbs the symmetry of the compensated solenoidal field of DNA. Instead of flowing in the internal region of DNA, some of the magnetic lines are forced to pass through the external region. In conditions of chromosome recombination in vivo, the read-out process may occur often than in the regulated cell cycle. Then the emitted sequence of entangled photons from one chromosome could be directly induced in the other. This may influence their common position and orientation. At the same time, the magnetic field of the cascade energy transfer through the bonding path of the DNA strand is not compensated in the triplex region. When combining with a similar region from another chromosome, the complex magnetic field may become compensated and stable.

Electronic properties of DNA: This subject is of increased interest to physicists and chemists. Even though the subject is far from being new, it is very controversial. One overview paper about this topic is presented by Dekker in Physics World [11]. The experimental results range from that of a good insulator to a good conductor, while many researchers think that DNA is able to provide some kind of energy flow. Currently two possible mechanisms of charge transfer are assumed: a coherent process of single step electron tunnelling, and a thermal hopping. It is interesting that a signature of cascade transfer exists in both suggested processes. While this theoretical mechanism seems reasonable, direct electrical measurements by a number of physics groups provide conflicting results. The results reported by Hans-Werner Fink and Christian Schonenberger [12], for $1 \mu \mathrm{m}$ long DNA in vacuum indicate good conductivity. However, this contradicts the theoretical considerations where DNA is expected to behave as a semiconductor with a large energy gap between the valence and conduction bands. The experiments by Porath et al. [13], for example, on a particular type of DNA - poly(gG)-poly(dC) DNA - show that it behaves as a large gap semiconductor.

One important fact that may influence the experimental results are the various environmental conditions in which the experiments are performed. Additionally, in a number of experiments, the electronic properties are not directly measured but derived from transient absorption spectra.

Let us now explain the possible electric properties of DNA from the point of view of BSM-SG theory. To have a stable energy cascade transfer, the DNA must be in an appropriate environment $(\mathrm{pH}$, temperature, ATP) and be long enough. In normal environments, DNA is assumed to be negatively charged. This charge, however, is uniformly distributed, so it may not influence the energy cascade transfer. In that case, the stored ring energies are synchronized and have an appropriate chirality. For appropriately small voltages along the DNA, the electrons do not leave their spatially distributed orbits. For larger voltages, however, the proximity proton field defining their orbits is biased, and the valence electron could jump across the gap synchronously. This transition, however, is expected to interact with the rotating energy states of the rings. The signature of this interaction might be the effect of a "single step electron-tunnelling mechanism". It is evident that such conditions may occur only if a stable energy cascade transfer through the DNA strand exists, but the latter effect is possible only in suitable environments. 


\section{Hypothesis of the decoding process in some of the complexes aminoacyle-tRNA synthetases - tRNA}

General considerations and code analysis: The prototype of tRNA molecule is coded in the gene. The current estimate for the number of tRNA in animals and plants is up to 50. The typical shape of the tRNA is shown in Figure 9. The tRNA binds to the appropriate enzyme that bonds to its end one of the 20 amino acids corresponding to the anticodon. Aminoacyl-tRNA synthetase is a family of 20 enzymes whose structures are fairly well known. The enzyme usually recognizes the corresponding tRNA using the anticodon.

Let us analyse the coding of the amino acids based on the anticodons used by tRNA. Instead of anticodons we may use for convenience the most popular RNA codons, shown in Table 1.

In the general case, the anticodon code is directly obtainable from the corresponding codon code. The number of codons is 64 . One exclusion from that rule is the anticodon of Alanine, for which the three codes GCU, GCC and GCA are replaced by one codon CGI (I Ionosine). This reduces the total number of anticodons to 61 .

One of the evident features of the amino acid coding is the code redundancy. Most of the amino acids are coded by more than one codon. One additional surprise comes from genome analysis. Some organisms do not have genes for all twenty aminoacyl-tRNA synthetases, but they still use all twenty amino acids to build their proteins.

The synthetase mechanism of the Aminoacyl-tRNA synthetases involves two steps. In the first step, they form an aminoacyl-adenylate in which the carboxyl of the amino acid is linked to the alpha-phosphate of ATP by displacing pyrophosphate. In the second step, but only if a correct tRNA is bound, the aminoacyl group of the aminoacyleadenylate is transferred to 2' or 3' terminal $\mathrm{OH}$ of the tRNA. The second step is conditional. This means that if a wrong enzyme is bound to tRNA, the output will be zero (not charging the appropriate amino acids). If we assume that the process of binding the correct complex of enzyme - tRNA is random, then the number of possible combinations for all 20 amino acid codes is $2^{20}=1048576$. This means that such a number of tRNA and enzymes are produced, but the number is unrealistic. Another possible option is for the enzymes to recognize or at least to increase the probability of binding to the correct tRNA, in which case the above number is significantly reduced.

One of the currently discussed problems is how these enzymes (aminoacyle-tRNA synthetases) recognize 20 aminoacides. It is estimated that they admit intrinsically small number of errors - about 1 in 10,000 . The unmistakeably recognition of 20 different flavours looks as quite intelligent task for a biomolecular structure of only few thousands atoms. This enigmatic feature is known as 20 different flavours of these enzymes. Such task could not be resolved unless the enzymes have some sensors for preliminary detection before binding and also some memory. Keeping in mind that the enzyme contains only a few thousands atoms, it is apparent that this capability should be built very economically using some basic physical properties at the atomic and molecular levels. Then the sensing should be based on some simplified detection mechanism of energy states, while the memory should be based on basic physical states of a binary type. Let us concentrate firstly on the memory feature. The possible binary physical states for a molecule of complexity of the enzymes are following:

(1) Quantum mechanical spin of the electron

(2) direction of magnetic field (S-N or N-S)
(3) chirality of rotating energy states (according to BSM-SG)

(4) electric charge ( + and -).

Option (4) could be excluded because its realization requires a complex structure of the semiconductor type. The most plausible option is (3) with some combination of options (1) and (2).

Let us calculate the required memory for decoding the codon of any amino acid using Boolean algebra without minimization. Any codon is a three-digit code. Any one of these digits needs 4 states to represent one of the four letters $(A, B, C, D)$. Then the three-digit code of the codon could be represented by a 12 bit binary code. This corresponds to a memory map of 4096 bits. Such a memory can hardly be achieved by any enzyme whose molecule usually includes only a few thousands atoms. Additionally, the enzyme must have remote sensors for recognising the tRNA before binding and tools for testing the anticodon.

Obviously, the above mentioned general approach for decoding of 20 flavours is not realistic. We must look for some code hidden in codons. Let us analyse the redundancy using some of the uncovered properties of the involved ring structures and the possibility of detecting their type. The purines are distinguishable from the pyrimidines by the number of atomic rings able to carry different stored energies, so they could be sensed by some kind of binary test. The possible mechanism of this test will be discussed later. To see how consecutive tests of the first, second and third letters of the codon will lead to some results, we make the following substitutions of some letters in the codons shown in Table 1. (the symbol $\rightarrow$ means a substitution)

$$
\text { Purines }(A \text { and } G) \rightarrow 2
$$

Pyrimidines $(\mathrm{C}$ and $\mathrm{U}) \rightarrow 1$

The digits 1 and 2 in this notations correspond to the number of the atomic rings.

Table 2 shows the codes after using the substitutions (6) and (7). The amino acids are identifiable by the positions of the codes. In Table 2 , we see that the binary codes (with base states " 1 " and " 2 ") are in a very strict order.

It is evident that the distribution of the amino acid codes based on the distinguishing features between the purines and pyrimidines is quite uniform. Only the codes for start and stop deviate from the uniformity of the distribution. From this simple analysis, however, one useful conclusion could be made:

\begin{tabular}{|l|l|l|l|}
\hline 111 & 111 & 121 & 121 \\
111 & 111 & 121 & 121 \\
\hline 112 & 112 & 122 & 122 \\
\hline 112 & 112 & 122 & 122 \\
\hline 111 & 111 & 121 & 121 \\
111 & 111 & 121 & 121 \\
\cline { 3 - 3 } 112 & 112 & 122 & 122 \\
\hline 112 & 112 & 122 & 122 \\
\hline 211 & 211 & 221 & 221 \\
\hline 211 & 211 & 221 & 221 \\
\hline 212 & 212 & 222 & 222 \\
\hline 212 & 212 & 222 & 222 \\
\hline 211 & 211 & 221 & 221 \\
211 & 211 & 221 & 221 \\
\hline 212 & 212 & 222 & 222 \\
\hline 212 & 212 & 222 & 222 \\
\hline
\end{tabular}

Table 2: The triplets abc corresponding to the anticodons shown in Table 1 after the substitutions (6) and (7). 
Citation: Sargoytchev SS (2017) A New Method for Analysis of Biomolecules Using the BSM-SG Atomic Models. J Biom Biostat 8: 339. doi:10.4172/2155-6180.1000339

The number of possible combinations could be significantly reduced if initial binary detection of purines or pyrimidines is performed.

The purines and pyrimidines are distinguishable by the number of rings. It will be described later that their possible remote detection has some similarity with the process of DNA readout described in section 6 .

Let us denote the tRNA anticodon by the triplet abc, but using instead the corresponding codons of RNA. The following example shows the reduction of the possible combinations in three consecutive binary tests (purines or pyrimidines) applied to the codes of Table 3 .

Let the first test for $a$ of the triplet $a b c$ is: $a=1$. This means that all codons starting with 2 will be excluded from the following tests, while those starting with 1 will be included. Then only 32 from of all 64 combinations will be left. The result is illustrated in Table 4, where the excluded combinations are masked by a grey colour. (Note: the grey colour in the print may look only slightly shaded).

We see that by three consecutive tests the available combinations are reduced from 64 to 8 , while they address only 4 amino acids. If we assume that this test is performed prior to the binding of the enzyme to the tRNA, then the required combinations for a probable correct match are only $2^{4}=16$. Consequently a possible remote sensing will reduce the amount of the necessary previously needed number of $2^{20}$ by a factor of 65536 .

The additional tests for identification of the correct amino acids require distinguishing of Adenine from Uracil and Guanine from Cytozine for every letter of the triplet abc. The detection for this identification, however, could not be remote and perhaps is performed in the second step of the synthetase mechanism. It is known, for example, that in glutaminyl-tRNA synthetase with its tRNA, the enzyme firmly grips the anticodon, spreading the three bases widely apart. Let us assume that the enzyme keeps in its memory the binary

\begin{tabular}{|c|c|c|}
\hline Code from Table 2 & No. of coded amino acids & Additional coding \\
\hline 111 & 4 & \\
\hline 112 & 4 & \\
\hline 121 & 4 & \\
\hline 122 & 3 & Stop \\
\hline 211 & 4 & Start \\
\hline 212 & 5 & \\
\hline 221 & 4 & \\
\hline 222 & 4 & \\
\hline
\end{tabular}

Table 3: Let us see to how many amino acids every code from the Table 2 is related. The result is shown in Table 3.

\begin{tabular}{|c|c|c|c|}
\hline $\begin{array}{l}111 \\
111\end{array}$ & \multirow{3}{*}{$\begin{array}{l}111 \\
111 \\
112 \\
112\end{array}$} & $\begin{array}{l}121 \\
121\end{array}$ & $\begin{array}{l}121 \\
121\end{array}$ \\
\hline \multirow{2}{*}{$\begin{array}{l}112 \\
112\end{array}$} & & \multirow{2}{*}{$\begin{array}{l}122 \\
122\end{array}$} & 122 \\
\hline & & & 122 \\
\hline \multirow{4}{*}{$\begin{array}{l}111 \\
111 \\
112 \\
112\end{array}$} & \multirow{4}{*}{$\begin{array}{l}111 \\
111 \\
112 \\
112\end{array}$} & 121 & \multirow{4}{*}{$\begin{array}{l}121 \\
121 \\
122 \\
122\end{array}$} \\
\hline & & 121 & \\
\hline & & & \\
\hline & & 122 & \\
\hline 211 & \multirow{4}{*}{$\begin{array}{l}211 \\
211 \\
212 \\
212\end{array}$} & 221 & 221 \\
\hline 211 & & 221 & 221 \\
\hline 212 & & 222 & 222 \\
\hline 212 & & 222 & 222 \\
\hline 211 & 211 & 221 & 221 \\
\hline 211 & 211 & 221 & 221 \\
\hline 212 & 212 & 222 & 222 \\
\hline 212 & 212 & $\begin{array}{l}222 \\
222\end{array}$ & 222 \\
\hline
\end{tabular}

Table 4: Test result for $a=1$. results of the previous remote detection (purines or pyrimidines) for every letter. Then the following test after the enzyme has grabbed the anticodon (for every letter of the codon) must be also a binary test of a type: Adenine (A) or Guanine (G) and Cytozine (C) or Uracil (U). How could they be distinguished, while they have the same number of rings? When we examine their structure, we see that the larger ring of Adenine includes three bonds of single valence, while the larger ring of Guanine includes four bonds of single valence. The ring of Cytosine includes two bonds of second valence, while the ring of Uracil includes one bond of second valence. These differences of the ring structures may cause significant differences in their spectral emission-absorption capability. A very narrow temperature range will define a rather narrow range of the population of the excited states. This may simplify the task for recognizing the differences between $(A$ and $G)$ and $(C$ and $U)$ by their spectral signatures (Tables 5 and 6 ).

After all these binary tests are performed, it seems that the correct amino acid should be decoded. However, we must not forget that the letters abc must be read in a correct order (from the most to the less significant digit). If this rule is not observed, the following code pairs could not be distinguished:

UUA-AUU

UUC-CUU

UUG-GUU

UCC-CCU

CCA-ACC

\begin{tabular}{|l|l|l|l|}
\hline 111 & 111 & 121 & 121 \\
\hline 111 & 111 & 121 & 121 \\
\hline 112 & 112 & 122 & 122 \\
\hline 112 & 112 & 122 & 122 \\
\hline 111 & 111 & 121 & 121 \\
\cline { 3 - 4 } 111 & 111 & 121 & 121 \\
\hline 112 & 112 & 122 & 122 \\
\hline 112 & 112 & 122 & 122 \\
\hline 211 & 211 & 221 & 221 \\
\hline 211 & 211 & 221 & 221 \\
\hline 212 & 212 & 222 & 222 \\
\hline 212 & 212 & 222 & 222 \\
\hline 211 & 211 & 221 & 221 \\
\hline 211 & 211 & 221 & 221 \\
\hline 212 & 212 & 222 & 222 \\
\hline 212 & 212 & 222 & 222 \\
\hline
\end{tabular}

Table 5: Test result for $b=2$.

\begin{tabular}{|l|l|l|l|}
\hline 111 & 111 & 121 & 121 \\
\hline 111 & 111 & 121 & 121 \\
\hline 112 & 112 & 122 & 122 \\
\hline 112 & 112 & 122 & 122 \\
\hline 111 & 111 & 121 & 121 \\
111 & 111 & 121 & 121 \\
112 & 112 & 122 & 122 \\
\cline { 3 - 4 } 112 & 112 & 122 & 122 \\
\hline 211 & 211 & 221 & 221 \\
\hline 211 & 211 & 221 & 221 \\
\hline 212 & 212 & 222 & 222 \\
\hline 212 & 212 & 222 & 222 \\
\hline 211 & 211 & 221 & 221 \\
\hline 211 & 211 & 221 & 221 \\
\hline 212 & 212 & 222 & 222 \\
\hline 212 & 212 & 222 & 222 \\
\hline
\end{tabular}

Table 6: Test results for $\mathrm{c}=2$. 


\section{AAG-GAA}

\section{UGG-GGU}

Assuming that the memory map of the enzyme is naturally minimized, the correct code read-out obviously must be initially detected prior to the remote sensing described above. To find the possible detection mechanism for the correct code readout direction, we may examine the structure of tRNA that has been already shown in Figure 9. In the left side of the figure, the real shape of the backbone structure is shown. In the right side of the same figure, the tRNA is presented as a flat curve to show more clearly some of its structural features, such as the folding of the single strand in a cloverleaf shape, the position of loops, the H-bonds, the position of the anticodon and the asymmetrical ends.

The tRNA strand is similar as the DNA strand and contains regularly attached $(\mathrm{O}+4 \mathrm{C})$ rings. In an appropriate environment, the tRNA could be energized, so a cascade energy transfer may occur through its strand in a way similar to that in DNA and proteins. The asymmetrically terminated end of tRNA may assure the appropriate direction of the cascade energy transfer. If such process is stable enough for a short time, the rotating energies in $(\mathrm{O}+4 \mathrm{C})$ rings can be synchronized, obtaining in this way an appropriate chirality. The same is true for the H-bonding base pairs and consequently for the codon loop. While the tRNA is not as long (75-90 nucleotides) the energy cascade process could not be as stable as in DNA and the proteins. The disruption of the energy cascade will lead to synchronized read-out of the rotating energy states in $(\mathrm{O}+4 \mathrm{C})$ rings that could be emitted as a coherent sequence of photon pulses whose parameters (phase sequence) will carry the direction of codon read-out. This read-out of $(\mathrm{O}+4 \mathrm{C})$ energies from its side might provoke a read-out process from the base pairs in a way similar to that in DNA. The conditions for emission from tRNA, however, are different from those of DNA due to the different shape of the tRNA molecule. Let us consider a hypothetical axis (not exactly linear), defined by the twisting shape (known as a secondary structure) of tRNA and passing from the anticodon loop of tRNA to its terminating ends. The two major side loops of tRNA could be regarded as approximately symmetrical pairs relative to the introduced axis. The supercoiling and tertiary structure may also influence the symmetry of the mentioned pairs, but will not influence the symmetrical features of the anticodon loop. At the same time, all the loops are free of $\mathrm{H}$-bonds. If we regard the emission as an EM field, it is evident that the two side loops are complementary and will have a comparatively smaller EM emission capability (because the magnetic lines appear closed in a circle around the introduced tRNA axis). The anticodon loop, however, does not have complementary symmetry with another loop, so its emission capability could be much larger. Then the emitted radiation from the anticodon loop could be detected remotely by the correct enzyme. The burst of the photon sequence from $(\mathrm{O}+4 \mathrm{C})$ rings read-out may serve as a synchronization that prepares the enzyme for detection of the anticodon. In that process the detection could be of a synchronous type, so it could allow an increased probability for detection of the anticodon sequence. The tRNA could be re-energize and the read-out sequence could be performed a few times. In this way, the anticodon could be detected by a number of corresponding enzymes.

The remotely detected sequence from anticodon loop (carrying information for purines or pyrimidines) could be regarded as very fast consecutive tests of the letters in the triplet abc prior to binding of the correct enzyme to tRNA. The test results, however, will be additionally needed for the consecutive tests (A or $\mathrm{G}$ and $\mathrm{C}$ or $\mathrm{U}$ ) that will be performed after the binding of the enzyme to tRNA. Let us assume that these test results are directly passed on to some kind of binary decoder implemented in the enzyme. Then after this remote test, the number of codons is reduced from 64 to 8 , corresponding to 4 amino acids. This significantly increases the probability of correct binding between tRNA and the corresponding enzyme.

Decoding algorithm: Figure 14 illustrates the decoding algorithm according to the analysis and considerations discussed in the previous

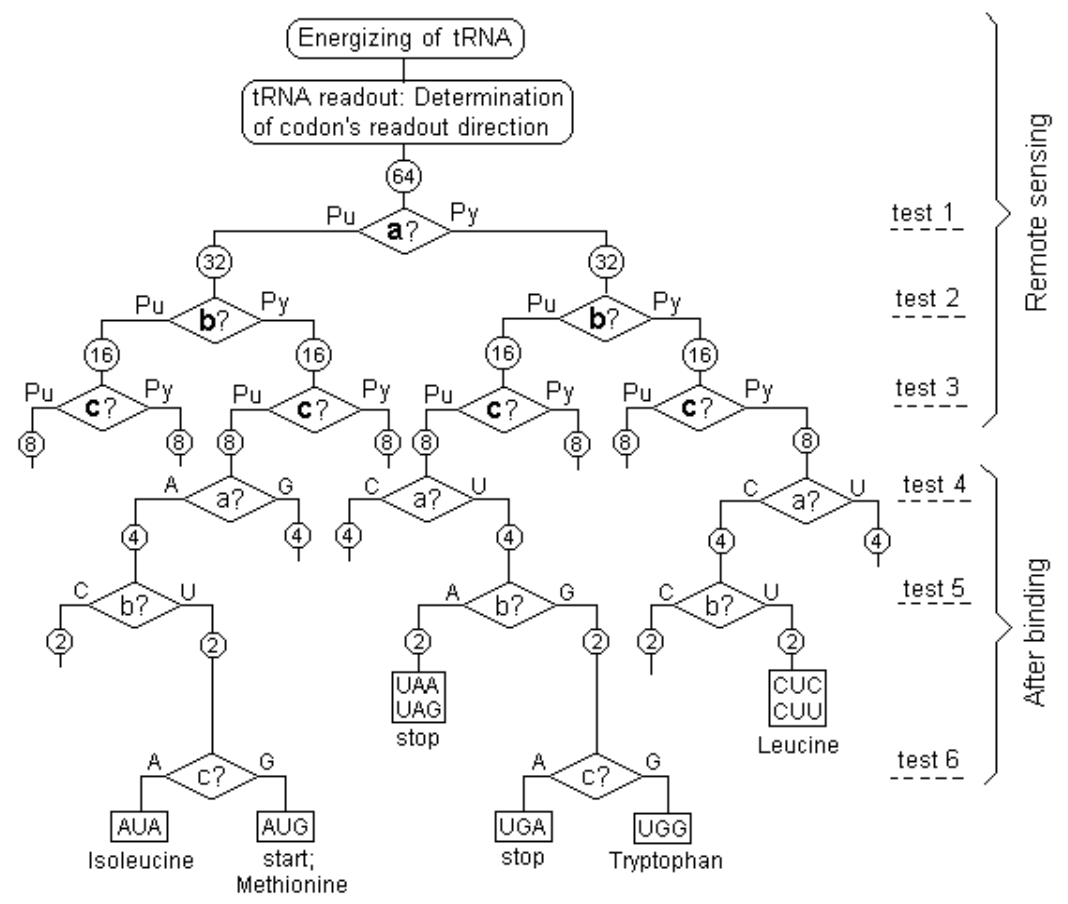

Figure 14: Decoding algorithm according to the analysis and considerations discussed in section 8 . 
Citation: Sargoytchev SS (2017) A New Method for Analysis of Biomolecules Using the BSM-SG Atomic Models. J Biom Biostat 8: 339. doi:10.4172/2155-6180.1000339

paragraph. Remote sensing includes tests $1,2,3$, while the other tests are performed after the tRNA is bound to the correct enzyme. The numbers in circles show the enabled codon combinations after each test. Not all the decoding tree is shown, but the algorithm for a possible decoding of all 20 aminoacids is the same. It is evident that a maximum of 6 tests is necessary for decoding any one of the aminoacids, but some of them are decoded even at test No 5. (Leucine, for example). Two of the stop codons are also decoded at test No. 5. This provides the opportunity to use test No. 6 for an additional truth test that should increase the decoding reliability. The other stop codon UGA, however, is decoded after test No. 6 . Then it could be interesting to study the statistics of UAA and UAG stop codons in comparison with the UGA stop codon (in both the RNA and DNA sequences).

According to the suggested algorithm, the tests from remote sensing increase the probability of correct binding between tRNA and the appropriate enzyme, but does not completely exclude wrong bindings. It is reasonable to expect that only a correct binding will lead to the attachment of amino acid after the tests 3, 4 and 5. A wrong binding will provide a zero result.

\section{Discussion}

It has been observed that biomolecules possess a stored energy. Analysis with the atomic models derived in the BSM-SG theory leads to a conclusion that DNA molecules and proteins possess an energy storage mechanism embedded in the atomic ring structures. This leads to a formulation of a hypothesis that the DNA might exhibit a specific EM signature with embedded codons sequence that is activated at the beginning of the replication. The new analytical tool shed a light on a possible communication between the DNA molecules residing in different cells. Together with the uncovered energy storage mechanism, this phenomenon shed light on some hitherto unknown processes in living organisms.

Further investigation using the described methodology might help to understand:

- How does the organism recognise a foreign living cell by their DNA EM signature?
- How the organism recognises and reacts against an implanted foreign tissue? phase?

- How the organism recognises and kills cancer cells at the initial

Brain proteins contain an enormous number of ring structures able to store energy. Appropriate synchronization of this energy may generate entangled quantum waves carrying coded information.

\section{Acknowledgement}

I would like to thank prof. Gordon Shephered from York University, Toronto for helpful comments and suggestions about the development of my theoretical models.

\section{References}

1. Sarg S (2002) A new approach for building of unified theory about the Universe and some results.

2. Sarg S (2005) Basic Structures of Matter-Supergravitation Unified Theory.

3. Sarg S (2011) Atlas of Atomic Nuclear Structures.

4. Sarg S (2014) Graphical 3D Modelling and Analysis of Molecules and Nanostructures. General Science Journal.

5. Sarg S (2003) A Physical Model of the Electron According to the Basic Structures of Matter Hypothesis. Physics essays 16: 180-195.

6. Anfinsen CB, Edsall JT, Richards FM (1981) Advances in protein chemistry. Academic Press.

7. Gelbart WM, Bruinsma RF, Pincus PF, Parsegian VA (2000) DNA-inspired electrostatics. Physics Today.

8. Saenger W (1984) Principles of Nucleic Acid Structure. Springer-Verlab, New York.

9. Lakowicz JR, Shen B, Gryczynski Z, D'Auria S, Gryczynski I (2001) Intrinsic fluorescence from DNA can be enhanced by metalic particles. Biochemical and Biophysical Re Comm 286: 875-879.

10. Kohwi Y, Panchenko Y (1993) Transcription-dependent recombination induced by triple-helix formation. Genes Dev 7: 1766-1778.

11. Dekker C (2001) Physics World.

12. Fink HW, Schonenberger C (1999) Electrical conduction through DNA molecule. Nature.

13. Porath D (2000) Direct measurement of electrical transport through DNA molecules. Nature. 